\title{
INTERAÇÃO GENÓTIPO AMBIENTE EM PROVAS DE GANHO EM PESO DE OVINOS CONFINADOS E A PASTO
}

\author{
Concepta Margaret McManus ${ }^{1}$, Rosana de Paula Branquinho ${ }^{2}$, Helder LouvandinI ${ }^{3}$, \\ SAMUEl REZENDE PAIVA ${ }^{4}$, BRUNO STEFANO DALlaGo ${ }^{5}$, ClÁUdia DAMO BERTOLI ${ }^{6}$ \\ ${ }^{1}$ Professora Doutora da Universidade Federal do Rio Grande do Sul, Porto Alegre, RS, Brasil - concepta.mcmanus@ufrgs.br \\ ${ }^{2}$ Graduada em Medicina Veterinária pela Universidade de Brasília, Brasília, DF, Brasil \\ ${ }^{3}$ Professor Doutor do Centro de Energia Nuclear na Agricultura, Uiversidade de São Paulo, Piracicaba, SP, Brasil. \\ ${ }^{4}$ Pesquisador Doutor da Empresa Brasileira de Pesquisa Agropecuária, Centro Nacional de Pesquisa de Recursos Genéticos e \\ Biotecnologia, Brasília, DF, Brasil \\ ${ }^{5}$ Professor Doutor da Universidade Paulista e responsável técnico pelo Laboratório de Doenças Infecciosas de Notificação \\ Obrigatória da Universidade de Brasília, Brasília, DF, Brasil. \\ ${ }^{6}$ Professora Mestra do Instituto Federal Catarinense, Camboriú, SC, Brasil.
}

\begin{abstract}
Objetivou-se com este trabalho verificar a existência de interação genótipo ambiente e o desempenho de ovinos mantidos em confinamento e a pasto. Foram utilizados oitenta e dois borregos da raça Santa Inês de 75 a 135 dias de idade. O período de adaptação foi 22 dias. Oriundos de cinco fazendas diferentes, 30 animais permaneceram 112 dias a pasto e 52 animais permaneceram 85 dias sob regime de confinamento. As características mensuradas nos animais foram ganho em peso(GP), comprimento do corpo (CC), perímetro torácico (PT), altura da anca (AA), altura do peito (AP), área de olho de lombo (AOL) e espessura de gordura por ultrassom (EG), perímetro escrotal (PE), número ovos/grama de fezes (OPG) e

escore corporal (EC). Um índice (IPGP) foi criado incorporando as características medidas. O software SAS ${ }^{\circledR}$ foi utilizado para as análises estatísticas. Houve diferença entre os animais para o tipo de teste (confinamento ou pasto) para as características GP, AOL, EG, PE e IPGP. Os animais em confinamento apresentaram resultados superiores aos do pasto para as características de ganho em pesoe inferiores para o IPGP e PE. A idade dos animais no início do teste não mostrou influencia sobre a expressão das características. Não houve evidência de interação genótipo x ambiente para animais da raça Santa Inês nas provas de ganho em pesoa pasto e em confinamento para as características avaliadas.
\end{abstract}

PALAVRAS-CHAVE: área de lombo; avaliação subjetiva; espessura de gordura; índice; ultrassom.

\section{GENOTYPE-ENVIRONMENT INTERACTION IN PERFORMANCE TESTS FOR SHEEP IN CONFINEMENT AND ON PASTURE}

\section{ABSTRACT}

This study aimed to verify the existence of genotypeenvironment interaction and performance of Santa Inês sheep in confinement and on pasture. Eighty-two Santa Ines lambs at 75-135 days of age were used. The adaptation period lasted 22 days. Animals were from five different farms, 30 animals were kept on pasture for 112 days and 52 animals were kept in confinement for 85 days. Animals were evaluated on weight gain (WG), body length (BL), thoracic perimeter (TP), hip height $(\mathrm{HH})$, breast height $(\mathrm{BH})$, rib-eye area (REA) and fat thickness (FD) using ultrasound, and scrotal circumference (SC), worm load (FEC) and body condition score (CS). An index (FI) was calculated using the measures taken. The SAS ${ }^{\circledR}$ software was used for statistical analysis. There was a statistical difference among animals for type of test (confinement or pasture) for WG, REA, FD, SP as well as FI. Measures for animals in confinement were generally higher than for animals on pasture, except for FI and SP. The age at the start of the test did not influence the expression of characteristics. There was no evidence found of a genotype-environment interaction for Santa Ines breed animals in weight gain on pasture and in confinement for the evaluated characteristics.

KEYWORDS: fat thickness; index; rib eye area; subjective evaluation; ultrasound.

Ci. Anim. Bras., Goiânia, v.13, n.2, p. 213-220, abr./jun. 2012 


\section{INTRODUÇÃO}

Quando a expressão de determinado genótipo depende do ambiente onde ele é avaliado dizemos que há interação entre o genótipo e o ambiente (EUCLIDES FILHO, 1999). Genótipos de raças altamente produtivas podem não se expressar totalmente em ambientes onde são submetidos a estresse ambiental. Fatores de estresse ambiental também podem ser características de interesse na produção animal como tolerância a carrapatos, endoparasitas, forragem de baixa qualidade e nutrição altamente variável em termos de quantidade ou qualidade, bem como temperatura ambiental (MCMANUS et al., 2009).

Independente das condições em que a ovinocultura é praticada há necessidade cada vez maior de lucratividade, maximizando o retorno dos recursos aplicados (REIS \& LOBO, 1991). O estabelecimento de um sistema de criação economicamente viável em determinada região requer a escolha de raças ou variedades que sejam adequadas às condições ambientais locais (MCMANUS et al., 2011).

Dependendo da intensidade das interações entre o genótipo e o ambiente, animais com potencial para rendimentos elevados em um ambiente podem ter comportamento diferenciado em outro sistema de criação. $\mathrm{O}$ animal considerado adequado para expressar seu potencial genético em determinado ambiente não será, necessariamente, o mais adequado em termos produtivos, mas, sim, econômicos. Alguns animais são menos adaptados ao seu ambiente e tendem a não se reproduzir tão eficientemente quanto os outros (MARIANTE et al., 2003). Dessa forma, indivíduos menos adaptados produzem menos descendentes, alterando a frequência gênica relativa nas gerações seguintes, que tenderão a apresentar animais melhor adaptados àquele ambiente específico.

Pesquisas revelam que a seleção praticada nos melhores ambientes, sem ou com poucos fatores estressores, tende a selecionar genótipos altamente produtivos (MADALENA, 2007). Em contraste, a seleção em ambientes adversos leva à seleção de animais menos produtivos, porém mais adaptados. De acordo com PERTOLDI et al. (2007), há a tendência de as respostas se associarem negativamente com as médias fenotípicas nos ambientes de baixa qualidade e positivamente com essas médias, nos ambientes de alta qualidade.

O objetivo deste trabalho foi verificar o desempenho e a existência de interação genótipo $\mathrm{x}$ ambiente de ovinos da raça Santa Inês sob regime de confinamento ou pastagem.

\section{MATERIAL E MÉTODOS}

Foram realizadas duas provas de ganho em pesocom ovinos da raça Santa Inês no Centro de Manejo de Ovinos da Fazenda Água Limpa da Universidade de Brasília, no Distrito Federal-DF. As provas foram gerenciadas por um comitê técnico local, composto de um Responsável Técnico, um Melhorista, um Nutricionista e um Médico Veterinário. Foram elegíveis borregos selecionados com base nas características desejadas na progênie. Aspectos como controle sanitário e ausência de defeitos nos animais foram verificados pelo comitê técnico local. Os animais apresentavam idade entre 75 - 135 dias, com diferença máxima de 45 dias entre os animais de um mesmo grupo. Verificou-se o controle genealógico dos animais, priorizando os realizados por programas de melhoramento, além das informações de data de nascimento, tipo de nascimento, peso ao nascer e regime alimentar em que foi criado o borrego. Os cordeiros tiveram origem em cinco rebanhos locais, filhos de nove reprodutores. Destes, oito tiveram filhos nos dois testes. As normas para os testes centrais em ovinos foram aprovados numa reunião no Ministério da Agricultura, Pecuária e Abastecimento (MAPA) em 2002, e estão de acordo com os usados em outros testes no Brasil.

No período de dezembro a abril, os animais foram mantidos em confinamento para a prova de ganho em pesoe alimentados com feno de coastcross (Cynodon dactylon (L.) pers.) ad libitum, sal mineral e $500 \mathrm{~g}$ de concentrado/animal/dia, constituído de 53\% milho triturado, 30\% farelo de soja, $8,4 \%$ farelo de girassol, $4,8 \%$ farelo de trigo, e $3,8 \%$ de minerais resultando num teor de $14 \%$ de proteína bruta e $72 \%$ de NDT, proporcionando um ganho médio de $200 \mathrm{~g} / \mathrm{animal} / \mathrm{dia}$.

No mesmo período, os animais foram mantidos a pasto (Panicum maximum v. Tanzânia), com suplementação de 200g/animal/dia do mesmo concentrado utilizado no confinamento, porém sem o núcleo mineral, que foi ofertado em separado ( $a d$ libitum), viabilizando ganho em peso médio de $110 \mathrm{~g} / \mathrm{animal} / \mathrm{dia}$.

Antes do início das provas, o comitê técnico local observou as condições sanitárias e as possíveis principais enfermidades (ectima contagioso, piolhos, lifadenite caseosa etc.) em todos os animais. Após a inspeção, os animais 
foram submetidos às vacinações e everminações estratégicas. Durante a prova, a cada 15 dias, foi realizado controle de endoparasitas por exames de fezes (ovos por grama de fezes - OPG) para avaliar o grau de infecção de cada animal. Também foi feita a contagem de Strongyloidea, Strongyloides e Moniezia a cada 15 dias. As Eimerias foram classificadas de acordo a sua ocorrência (0 a 4). O período de adaptação foi de 22 dias. A fase do teste teve duração de 85 dias para o confinamento $(n=52)$ e 112 dias a pasto $(n=30)$.

Os animais foram avaliados quanto ao ganho em peso(GP), comprimento do corpo (CC), perímetro torácico $(\mathrm{PT})$, altura da anca (AA), altura do peito (AP), área de olho de lombo (AOL) e espessura de gordura por ultrassom (EG), perímetro escrotal (PE), ovos por grama de fezes (OPG) e escore corporal (EC). Um índice foi criado incorporando as características medidas e calculado da seguinte forma: IPGP (índice da prova de ganho de peso) $=$ ganho em pesomédio por dia $(40 \%)+$ área de olho de lombo $(15 \%)+$ perímetro escrotal $(10 \%)+$ espessura de gordura $(5 \%)+$ escore visual (30\%). Antes da confecção final dos índices, as medidas objetivas tiveram ajuste linear para idade.

$\mathrm{O}$ escore visual foi composto pelos escores de conformação $(\mathrm{C})$, precocidade $(\mathrm{P})$, musculatura (M), tipo racial (TR) e aprumos (A), cada item contribuindo com 20\% do escore visual, representando $6 \%$ do IPGP. A escala de escore visual variou de um a seis, onde seis foi maior grau. Essa escala foi relativa ao padrão zootécnico do grupo que estava sendo avaliado; portanto, em todos os grupos os animais receberam notas de 1 a 6 , independente de qualquer comparação absoluta com outro grupo. $\mathrm{O}$ escore visual nota seis representa um animal excelente, 5 - ótimo, 4 bom, 3 - médio, 2 - inferior e 1 representa um animal muito inferior.

$\mathrm{Na}$ avaliação visual da conformação (C) foram levadas em conta características como desenvolvimento de massa muscular e quantidade total estimada da porção comestível da carcaça, valorizando-se aspectos estruturais, dentre eles o porte e harmonia das características morfofuncionais.

A precocidade (P) foi avaliada pela capacidade ou grau de deposição precoce de gordura, buscando animais que atinjam a terminação (acabamento para o abate) mais cedo. $\mathrm{O}$ biótipo do animal também foi analisado. O tipo longilíneo é alto com pouca profundidade de costela e se caracteriza como um animal mais tardio (notas mais baixas), enquanto o de estrutura mediolíneo apresenta-se com boa profundidade de costela, boa massa muscular, virilha preenchida desde que aliada a um bom desenvolvimento corporal, definindo o animal mais precoce, recebendo notas mais altas para esta característica.

A característica musculatura (M) foi avaliada pela observação de pontos específicos no animal, como antebraço, membros anterior e posterior e profundidade da garupa. As notas foram dadas analisando os animais parados e em movimento. Quando parados, os animais que apresentam os membros afastados, tanto de frente como por trás mostram musculatura mais desenvolvida. Quando se deslocam, o movimento dos músculos, que se contraem e aumentam de volume ritmicamente, delineando sua forma, diferenciam músculo de gordura, que não tem sustentação e forma definida. Animais mais musculosos receberam notas mais altas.

O tipo racial (TR) foi avaliado quanto ao conjunto de atributos raciais preconizados no padrão oficial da raça Santa Inês (cabeça, pelagem, pigmentação da pele, entre outras).

Os aprumos foram avaliados em relação à proporção, direção e articulações dos membros anteriores e posteriores.

Os dados foram analisados usando-se o software $\mathrm{SAS} \AA$, por meio dos procedimentos GLM, CORR e PRINCOMP. O modelo estatístico utilizado foi:

$$
\mathrm{Y}_{\mathrm{ijklm}}=\mathrm{T}_{\mathrm{i}}+\mathrm{R}_{\mathrm{j}}+\mathrm{TR}_{\mathrm{ij}}+\mathrm{P}_{1}+\mathrm{IIT}_{\mathrm{m}}+\mathrm{E}_{\mathrm{ijklm}}
$$

Em que $Y_{\mathrm{ijklm}}$ é a característica medida no animal, $T_{i}$ é o efeito fixo do Teste $(i=1,2), R_{j}$ é o efeito fixo do Reprodutor $(\mathrm{j}=8), \mathrm{TR}_{\mathrm{ij}}$ é o efeito fixo da interação entre reprodutor e fazenda, $\mathrm{P}_{1}$ é o efeito fixo de Tipo de parto $(1=2)$, IIT $_{\mathrm{m}}=$ efeito da covariável Idade no Início do Teste e $\mathrm{E}_{\mathrm{ijk} k \mathrm{~m}}$ é o efeito aleatório do erro.

Os dados de OPG foram transformados em $\log (x+10)$ e os de escore foram transformados usando raiz quadrada.

\section{RESULTADOS E DISCUSSÃO}

Pelos dados apresentados na tabela 1, verificou-se que, em geral, houve baixa variação para as características mensuradas, exceto as de OPG, que foram muito altas. Isso era esperado, uma vez que houve um período de adaptação quando os animais entraram nas provas para eliminar os efeitos ambientais das fazendas de origem (BIF, 1986). 
TABELA 1. Características das provas de ganho em pesode ovinos Santa Inês no Distrito Federal

\begin{tabular}{|c|c|c|c|c|c|c|c|c|c|c|c|}
\hline Carac. & $\mathbf{N}$ & Média & DP & Min. & Max. & Carac. & $\mathbf{N}$ & Média & DP & Min. & Max. \\
\hline Sdea & 81 & 519 & 1368 & 0 & 8200 & C & 79 & 4 & 0,84 & 3 & 6 \\
\hline Sdes & 81 & 20 & 71,45 & 0 & 450 & $\mathbf{P}$ & 79 & 4 & 0,87 & 2 & 6 \\
\hline Mon & 81 & 13 & 106 & 0 & 950 & $\mathbf{M}$ & 79 & 4 & 0,84 & 2 & 6 \\
\hline Eim & 81 & 0,27 & 0,59 & 0 & 3 & TR & 79 & 4 & 1,33 & 1 & 6 \\
\hline GPN_I & 81 & 0,14 & 0,06 & 0,04 & 0,32 & $\mathbf{A}$ & 79 & 4 & 0,7 & 3 & 6 \\
\hline GPN_F & 79 & 0,13 & 0,03 & 0,08 & 0,2 & IPGP & 79 & 9 & 1,68 & 4,26 & 12,97 \\
\hline GPI_F & 79 & 0,16 & 0,07 & 0,05 & 0,31 & $\mathrm{CC}$ & 28 & 64 & 4,52 & 56 & 72 \\
\hline AOL & 79 & 6 & 1,89 & 1,74 & 10,18 & PT & 28 & 74 & 5,1 & 64 & 83 \\
\hline EG & 79 & 0,28 & 0,11 & 0,1 & 0,6 & $\mathbf{A A}$ & 28 & 70 & 4,39 & 62 & 78 \\
\hline PE & 76 & 26 & 4,33 & 16 & 35 & & & & & & \\
\hline
\end{tabular}

Carac. - Característica avaliada; N - número de avaliados; DP - Desvio Padrão; Min. valor mínimo; Max - valor máximo; Sdea Strongyloidea (n); Sdes - Strongyloides(n); Mon - Moniezia(n); Eim - Eimeria(n); GPN_I - ganho em pesodo nascimento ao início da prova (Kg/dia); GPN_F - ganho em pesodo nascimento ao final da prova (Kg/dia); GPI_F - ganho em pesodo início ao final da prova $(\mathrm{Kg} / \mathrm{dia}) ; \mathrm{AOL}$ - área de lombo $\left(\mathrm{cm}^{2}\right) ; \mathrm{EG}$ - espessura de gordura $(\mathrm{cm}) ; \mathrm{PE}$ - perímetro escrotal $(\mathrm{cm}) ; \mathrm{C}$ - conformação (escores 1-6); P - precocidade (escores 1-6); M - musculatura (escores 1-6); TR - tipo racial (escores 1-6); A - aprumos e membros (escores 1-6); IPGP - índice da prova de ganho de peso; CC - comprimento do corpo (cm); PT - perímetro torácico (cm); AA altura da anca $(\mathrm{cm}) ; \mathrm{AP}$ - altura do peito $(\mathrm{cm})$.

A Tabela 2 mostra a análise de variância para as características mensuradas nas duas provas de ganho de peso. Houve diferença entre as duas provas para as características GPN_I, GPN_F, GPI_F, AOL, EG, IPGP e PE $(\mathrm{P}<0,001)$, EIM $(\mathrm{P}<0,05)$ e não foi possível detectar diferenças para as características Sdea, Sdes, Mon e Escores Visuais (C, P, M, TR e A). Os animais em confinamento apresentaram crescimento corporal mais rápido, mas os animais a pasto apresentaram maiores PE. Os ovinos a campo terminaram a prova com idade superior (aproximadamente dois meses) e, de acordo com o peso dos testículos dos animais, mais próximos da puberdade, embora o peso vivo dos animais no final dos testes tenham sido similares. De acordo com ALVES et al. (2006), o crescimento dos testículos e a puberdade estão mais relacionados com a alimentação dos animais do que a idade. No experimento desses autores, todos os animais foram criados a pasto e, na época das chuvas, não receberam suplementação, que diminui $o$ desenvolvimento dos mesmos.

A taxa de crescimento em animais jovens é, em grande parte, uma função do apetite, capacidade de crescimento dos tecidos (ósseo e muscular) e eficiência da manutenção corporal. Em baixos níveis de ração, a variação na taxa de crescimento é principalmente afetada pela manutenção corpórea requerida. Em altos níveis, manutenção e capacidade de crescimento dos tecidos são os que mais contribuem (BERG \& BUTTERFIELD, 1979). No Brasil, o uso de cruzamentos entre raças especializadas e as raças adaptadas tem sido uma das alternativas dos produtores na busca de animais mais produtivos nas condições de ambiente tropical e subtropical. Na maioria dos países em desenvolvimento localizados nos trópicos, o baixo desempenho reprodutivo, tanto de raças nativas quanto importadas, resultou da combinação de genética, manejo e fatores ambientais (AGYEMANG et al., 1991). 
TABELA 2. Resultados da ANOVA para características de ovinos Santa Inês em provas de ganho em pesono Distrito Federal

\begin{tabular}{|c|c|c|c|c|c|c|c|c|c|c|c|c|c|c|c|c|c|c|c|c|}
\hline & Sdea & Sdes & Mon & Eim & \begin{tabular}{|c|} 
GPN_I \\
(Kg/dia)
\end{tabular} & $\begin{array}{l}\text { GPN_F } \\
(\mathrm{Kg} / \mathrm{dia})\end{array}$ & \begin{tabular}{|c|} 
GPI_F \\
(Kg/dia)
\end{tabular} & $\begin{array}{l}\mathrm{AOL} \\
\left(\mathrm{cm}^{2}\right)\end{array}$ & $\begin{array}{c}\text { EG } \\
(\mathrm{cm})\end{array}$ & $\mathrm{C}$ & $\mathrm{P}$ & M & TR & A & IPGP & $\begin{array}{c}\mathrm{PE} \\
(\mathrm{cm})\end{array}$ & $\begin{array}{c}\mathrm{CC} \\
(\mathrm{cm}) \\
\end{array}$ & $\begin{array}{l}\text { PT } \\
(\mathrm{cm})\end{array}$ & $\begin{array}{c}\text { AA } \\
(\mathrm{cm})\end{array}$ & $\begin{array}{c}\text { AP } \\
(\mathrm{cm})\end{array}$ \\
\hline Teste & ns & ns & ns & $*$ & $* * *$ & $* * *$ & $* * *$ & $* * *$ & $* * *$ & ns & ns & ns & ns & ns & $* * *$ & $* * *$ & . & . & . & $\cdot$ \\
\hline Reprodutor & $* *$ & $* * *$ & $*$ & ns & $* *$ & $*$ & $* * *$ & ns & ns & ns & ns & ns & ns & ns & ns & ns & $*$ & $*$ & ns & $* *$ \\
\hline Teste x Reprodutor & ns & $\mathrm{ns}$ & $\mathrm{ns}$ & $\mathrm{ns}$ & ns & ns & $\mathrm{ns}$ & $\mathrm{ns}$ & $\mathrm{ns}$ & ns & ns & ns & $\mathrm{ns}$ & ns & $\mathrm{ns}$ & $\mathrm{ns}$ & . & . & . & . \\
\hline Tipo de Nasc. & ns & $\mathrm{ns}$ & $\mathrm{ns}$ & ns & ns & ns & ns & $\mathrm{ns}$ & ns & ns & ns & ns & $\mathrm{ns}$ & ns & ns & ns & ns & $\mathrm{ns}$ & ns & $\mathrm{ns}$ \\
\hline Idade & ns & $\mathrm{ns}$ & $\mathrm{ns}$ & $\mathrm{ns}$ & $* *$ & $*$ & $\mathrm{~ns}$ & $\mathrm{~ns}$ & $\mathrm{~ns}$ & $\mathrm{~ns}$ & $\mathrm{~ns}$ & $\mathrm{~ns}$ & $*$ & $\mathrm{~ns}$ & $\mathrm{~ns}$ & $*$ & $\mathrm{~ns}$ & $\mathrm{~ns}$ & $\mathrm{~ns}$ & $\mathrm{~ns}$ \\
\hline $\mathrm{R}^{2}$ & 0,40 & 0,38 & 0,21 & 0,26 & 0,76 & 0,48 & 0,85 & 0,57 & 0,72 & 0,16 & 0,27 & 0,25 & 0,16 & 0,14 & 0,67 & 0,49 & 0,65 & 0,52 & 0,43 & 0,56 \\
\hline CV (\%) & 218,11 & 303,58 & 777,18 & 201,06 & 24,23 & 17,36 & 18,34 & 21,99 & 22,93 & 19,95 & 19,85 & 20,51 & 30,85 & 15,99 & 11,31 & 12,64 & 4,59 & 5,33 & 5,29 & 4,33 \\
\hline Média Geral & 519,14 & 19,75 & 12,96 & 0,27 & 0,14 & 0,13 & 0,16 & 6,04 & 0,28 & 4,13 & 4,01 & 3,80 & 4,23 & 4,37 & 9,13 & 26,31 & 64,32 & 73,79 & 69,75 & 70,64 \\
\hline \multicolumn{21}{|l|}{ Médias: } \\
\hline Confinamento & 0,00 & 0,00 & 0,00 & 0,00 & 0,08 & 0,11 & 0,23 & 4,44 & 0,40 & 4,00 & 3,71 & 3,54 & 4,25 & 4,29 & 7,45 & 24,52 & & & & \\
\hline Pasto & 824,50 & 31,37 & 20,59 & 0,43 & 0,18 & 0,01 & 0,11 & 6,92 & 0,21 & 4,20 & 4,18 & 3,94 & 4,22 & 4,41 & 10,05 & 27,24 & & & & \\
\hline
\end{tabular}

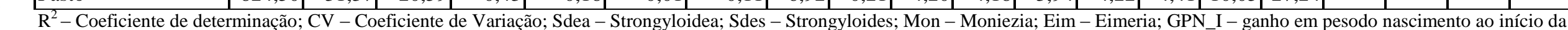
prova; GPN_F - ganho em pesodo nascimento ao final da prova; GPI F - ganho em pesodo início ao final da prova; AOL - área de lombo; EG - espessura de gordura; PE - perímetro escrotal; C - conformação; P - precocidade de acabamento; M - musculatura; TR - tipo racial; A - aprumos e membros; IPGP - índice da prova de ganho de peso; CC - comprimento do corpo; PT perímetro torácico; AA - altura da anca; $\mathrm{AP}$ - altura do peito. 
TABELA 3. Correlações fenotípicas (Pearson) entre as características de ovinos Santa Inês em provas de ganho em pesono Distrito Federal.

\begin{tabular}{|c|c|c|c|c|c|c|c|c|c|c|c|c|c|c|c|c|c|c|c|}
\hline & Sdea & Sdes & Mon & Eim & GPN_I & GPN_F & GPI_F & AOL & EG & $\mathbf{P E}$ & C & $\mathbf{P}$ & $\mathbf{M}$ & TR & $\mathbf{A}$ & IPGP & $\mathrm{CC}$ & PT & $\mathbf{A A}$ \\
\hline Sdes & $0,51^{1}$ & & & & & & & & & & & & & & & & & & \\
\hline Mon & 0,00 & $-0,03$ & & & & & & & & & & & & & & & & & \\
\hline Eim & 0,42 & 0,24 & $-0,06$ & & & & & & & & & & & & & & & & \\
\hline GPN_I & 0,26 & 0,14 & 0,03 & 0,18 & & & & & & & & & & & & & & & \\
\hline GPN_F & 0,04 & 0,01 & $-0,13$ & 0,16 & 0,83 & & & & & & & & & & & & & & \\
\hline GPI_F & $-0,32$ & $-0,21$ & $-0,17$ & $-0,26$ & $-0,64$ & $-0,24$ & & & & & & & & & & & & & \\
\hline $\mathrm{AOL}$ & $-0,07$ & $-0,07$ & $-0,14$ & 0,11 & 0,57 & 0,70 & $-0,32$ & & & & & & & & & & & & \\
\hline EG & $-0,36$ & $-0,29$ & $-0,19$ & $-0,25$ & $-0,51$ & $-0,17$ & 0,83 & $-0,22$ & & & & & & & & & & & \\
\hline PE & $-0,19$ & $-0,21$ & $-0,28$ & 0,19 & 0,28 & 0,57 & 0,08 & 0,65 & 0,06 & & & & & & & & & & \\
\hline $\mathrm{C}$ & $-0,12$ & $-0,15$ & $-0,15$ & 0,13 & 0,17 & 0,44 & 0,21 & 0,37 & 0,11 & 0,59 & & & & & & & & & \\
\hline $\mathrm{P}$ & $-0,17$ & $-0,20$ & $-0,13$ & 0,19 & 0,30 & 0,54 & 0,07 & 0,47 & 0,04 & 0,65 & 0,72 & & & & & & & & \\
\hline M & $-0,04$ & $-0,10$ & $-0,11$ & 0,19 & 0,30 & 0,53 & 0,12 & 0,46 & 0,05 & 0,57 & 0,62 & 0,65 & & & & & & & \\
\hline TR & 0,07 & 0,09 & $-0,01$ & 0,00 & 0,08 & 0,03 & 0,00 & $-0,01$ & $-0,03$ & $-0,21$ & $-0,10$ & $-0,15$ & $-0,19$ & & & & & & \\
\hline A & 0,10 & 0,13 & $-0,05$ & $-0,03$ & 0,10 & 0,05 & $-0,03$ & 0,07 & $-0,09$ & 0,06 & 0,18 & $-0,03$ & 0,19 & 0,28 & & & & & \\
\hline IPGP & 0,06 & 0,01 & $-0,12$ & 0,32 & 0,66 & 0,75 & $-0,39$ & 0,82 & $-0,36$ & 0,69 & 0,56 & 0,62 & 0,59 & 0,16 & 0,28 & & & & \\
\hline $\mathrm{CC}$ & & & & & $-0,27$ & 0,18 & 0,82 & 0,39 & 0,33 & 0,78 & 0,51 & 0,71 & 0,67 & $-0,13$ & 0,44 & 0,55 & & & \\
\hline PT & & & & & $-0,05$ & 0,34 & 0,73 & 0,49 & 0,49 & 0,56 & 0,64 & 0,67 & 0,70 & 0,27 & 0,36 & 0,76 & 0,59 & & \\
\hline AA & & & & & 0,00 & 0,40 & 0,74 & 0,53 & 0,41 & 0,69 & 0,66 & 0,69 & 0,65 & 0,08 & 0,55 & 0,72 & 0,67 & 0,67 & \\
\hline AP & & & & & $-0,09$ & 0,32 & 0,74 & 0,51 & 0,36 & 0,65 & 0,56 & 0,64 & 0,65 & 0,14 & 0,56 & 0,71 & 0,68 & 0,65 & 0,95 \\
\hline
\end{tabular}

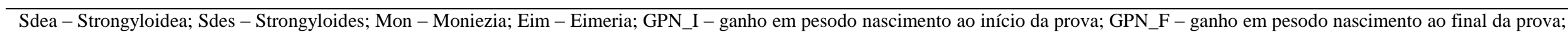
GPI F - ganho em pesodo início ao final da prova; AOL - área de lombo; EG - espessura de gordura; PE - perímetro escrotal; C- conformação; P - precocidade de acabamento; M musculatura; TR - tipo racial; A - aprumos e membros; IPGP - índice da prova de ganho de peso; CC - comprimento do corpo; PT - perímetro torácico; AA - altura da anca; AP - altura do peito.

${ }^{1}$ Nivel de significância da correlação: 0,00 a $<0,22$ NS; 0,22 a 0,29 $(\mathrm{P}<0.05)$ e $>0,29(\mathrm{P}<0,01)$. 


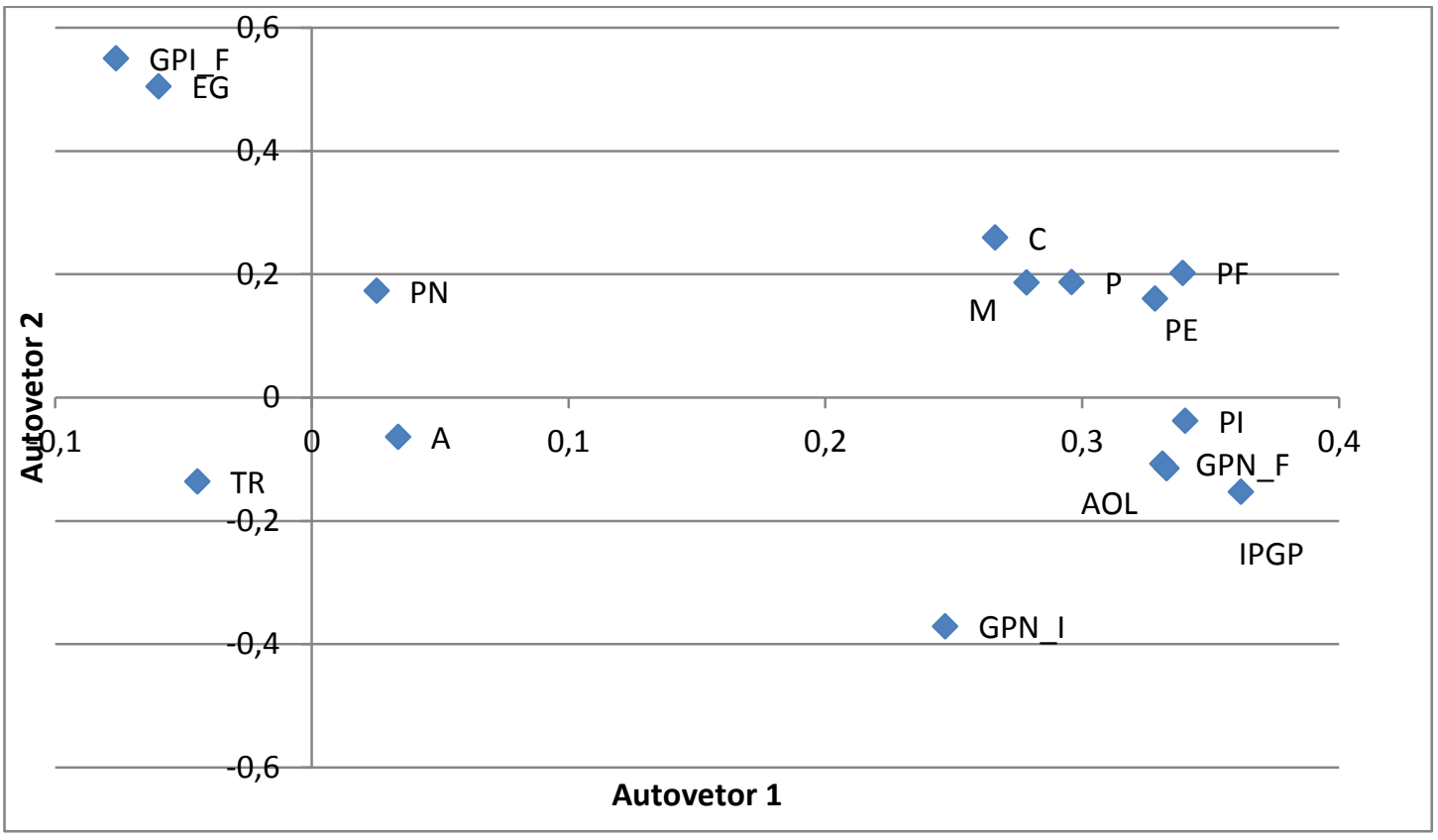

GPN_I - ganho de peso do nascimento ao início da prova; GPN_F - ganho de peso do nascimento ao final da prova; GPI_F - ganho de peso do início ao final da prova; AOL - área de lombo; EG - espessura de gordura; $\mathrm{PE}$ - perímetro escrotal; $\mathrm{C}$ - conformação; $\mathrm{P}$ - precocidade de acabamento; $\mathrm{M}$ - musculatura; $\mathrm{TR}$ - tipo racial; A - aprumos e membros; IPGP - índice da prova de ganho de peso; PN - peso ao nascer; PI - peso no início da prova; $\mathrm{PF}$ - peso ao final da prova.

FIGURA 1. Autovetores1 e 2 para características de ovinos Santa Inês em provas de ganho em pesono Distrito Federal.

As correlações em geral foram baixas entre OPG e médias entre ganhos de peso e escores visuais. Altas correlações foram encontradas entre as quatro características de tamanho (CC, PT, AA e AP) e peso (GPI_F) e média a altas entre tamanho e os três componentes dos escores visuais $(\mathrm{C}, \mathrm{P}$ e $\mathrm{M})$, porem, quando esses $(\mathrm{C}, \mathrm{P}$ e $\mathrm{M})$ foram contrastados com peso (GPI_F), essas correlações foram muito baixas (Tabela 3). Isto pode significar que o avaliador, sem perceber, deu notas mais altas aos animais de maior tamanho (altos CC, PT, AA e AP).

As correlações entre as medidas de tamanho (CC, PT, AA e AP) não foram calculadas, pois essas medidas só foram tomadas nos animais mantidos em confinamento e esses tiveram $\mathrm{OPG}$ zero, não permitindo esse cálculo.

Quatorze reprodutores foram testados, possuindo de 3 a 11 filhos cada um. Seis deles, com 6 a 11 filhos, tiveram produtos seus nos dois testes, promovendo os laços genéticos necessários para avaliação. Não foi detectada interação entre esses reprodutores e o tipo de prova (não mostrado). Isso significa que os filhos de reprodutores que foram melhores na prova em confinamento, também o foram na prova a pasto. Genótipos diferentes podem responder de várias formas dependendo do ambiente.
Os genótipos de animais altamente produtivos podem se expressar livremente se não houver estresse ambiental, enquanto genótipos selecionados tanto para características de sobrevivência e reprodução quanto para produção podem apresentar melhor rendimento quando submetidos a ambientes adversos (PERTOLDI et al., 2007). Estresse ambiental inclui os desafios a carrapatos, parasitos internos, forragem de baixa qualidade e nutrição altamente variável em termos de quantidade ou qualidade, bem como temperatura ambiental (McMANUS et al., 2010).

Nas duas provas, o ambiente foi favorável ao crescimento dos animais, o que pode explicar a falta de interação. As diferenças iniciais no crescimento dos animais, antes de participarem do teste (como efeito de tipo de nascimento ou idade), desapareceram ao final, mostrando que o período de adaptação foi adequado para remover diferenças devido à origem dos animais (KRAUSSLICH, 1974). Segundo ANDERSEN et al. (1981), o período de adaptação deve ser suficiente para permitir um crescimento compensatório para igualar as condições corporais dos animais. A idade dos animais é muito importante nesse período e esse tempo deve ser usado para eliminar os parasitas e igualar os níveis 
de vitaminas e minerais dos animais (ANDERSEN et al. 1981). O local de realização desse período de adaptação deve permitir fácil transferência dos animais para o local das provas.

Não foi possível detectar diferenças entre os testes, fazendas ou tipo de nascimento para os escores visuais (C, P, M, TR e A). Embora estes tenham um peso coletivo de $30 \%$ no índice final da prova (6\% cada um), o efeito das características objetivas (GP, AOL e PE) detectou diferenças no final dos dois testes, com animais criados a pasto apresentando um índice maior. Os animais que entraram no teste foram selecionados pelos fazendeiros de acordo com seus critérios.

$\mathrm{Na}$ análise dos componentes principais, o primeiro componente mostrou que, em geral, animais maiores para uma característica foram maiores para todas, como esperado (Figura 1). O segundo componente mostrou animais com alto ganho em pesodurante o teste, mas com AOL e IPGP baixos. Esse animal descrito pelo segundo componente não é interessante para o produtor. Os primeiros dois componentes explicaram $63 \%$ de toda a variação durante o teste. Mais uma vez os escores subjetivos (C, P, M e TR) acompanharam as medidas de tamanho (CC, PT, AA e AP), exceto aprumos e membros (A), que não está relacionado com o tamanho do animal, mostrando a dificuldade que existe na separação dessas medidas em relação ao tamanho do animal.

\section{CONCLUSÃO}

Não houve evidência de interação genótipo ambiente para animais da raça Santa Inês em provas de ganho em pesoem confinamento e a pasto, mostrando que um animal selecionado em um dos ambientes testados pode responder bem no outro. $\mathrm{O}$ uso de escores subjetivos não se mostrou eficiente na detecção de diferenças nos fenótipos dos animais.

\section{REFERÊNCIAS}

ALVES, J. M., MCMANUS, C., LUCCI, C. M, CARNERIO, H., DALLAGO, B. S., CADAVID, V. G., MARSIAJ, P., LOUVANDINI, H. Estação de Nascimento e Puberdade em Cordeiros Santa Inês. Revista Brasileira de Zootecnia, v. 35, n. 03, p. 958-966, 2006.

AGYEMANG, K; LITTLE, D. A.; BAH, M. L.; DWINGER, R. H. Effects of postpartum body weight changes on subsequent reproductive performance in N'dama cattle maintained under traditional husbandry systems. Animal Reproduction Science, v.26, p.51-59, 1991.

ANDERSEN, B.B., BAERDEMAEKER, A., BITTANTE, G., BONAITI, B., COLLEAU, J. J., FIMLAND, E., JANSEN, J., LEWIS, W. H. E., POLITIEK, R. D., SEELAND, G., TEEHAN, T. J. and WERKMEISTER, F. Performance testing of bulls in AI: Report of a working group of the Commission on Cattle Production. Livestock Production Science, v.8, p.101-119.1981

BERG, R.T.; BUTTERFIELD, R.M. Nuevos conceptos sobre desarrollo del ganado vacuno. Zaragosa: Acribia,.297p.1979.

BIF Guidelines for Uniform Beef Improvement (5 th Rev. Ed.) pp 8.1 - 8.6. Beef Improvement Federation, Raleigh, NC. 1986.

EUCLIDES FILHO, K. Melhoramento Genético Animal no Brasil. Fundamentos, História e Importância. Campo Grande: EMBRAPA. 86p. 1999.

KRAUSSLICH, H. General Recommendations on procedures for performance and progeny testing for beef characteristics. Livestock Production Science, v.1, p.33 45, 1974.

MADALENA, F.E. Efeitos colaterais da genética de alta produção.2007 http://www.milkpoint.com.br/?actA=7\&areaID=61\&secao $\underline{\mathrm{ID}=171 \& \text { noticiaID }=42598}$

MARIANTE, A.S., MCMANUS, C., MENDONÇA, J.F. Country report of Animal Genetic Resources: Brazil, EMBRAPA, Brasília,91p. 2003

MCMANUS, C. M. ; PINTO, B.F.; MARTINS, R.S.; LOUVANDINI, H. ; PAIVA, S.R.; BRACCINI NETO, J.; PAIM, T.P. Selection objectives and indices for hair sheep in central Brazil. Revista Brasileira de Zootecnia, v.40, p. 2713-2720, 2011.

McMANUS, C. M. ; PALUDO, G.R.; LOUVANDINI, H. ; GUGEL, R.; SASAKI, L.C.B.; PAIVA, S.R. Heat Tolerance in Brazilian Sheep: Physiological and Blood Parameters. Tropical Animal Health and Production, v. 9, p. 95-101, 2009

MCMANUS, C.; PAIVA, S.; ARAUJO, R.O. Genetics and breeding of sheep in Brazil. Revista Brasileira de Zootecnia, v.39, p.236-246, 2010.

PERTOLDI, C.; BIJLSMA, R.; LOESCHCKE, V. Conservation genetics in a globally changing environment: present problems, paradoxes and future challenges. Biodiversity and Conservation, v. 16, p. 4147-4143, 2007.

REIS, J.C., LOBO, R.B. Interações genótipo-ambiente nos animais domésticos, Gráfico e Editora F.C.A., Ribeirão Preto,194p., 1991 